\title{
Rank gradient and torsion groups
}

D. Osin *

\begin{abstract}
We construct first examples of infinite finitely generated residually finite torsion groups with positive rank gradient. In particular, these groups are non-amenable. Some applications to problems about cost and $L^{2}$-Betti numbers are discussed.
\end{abstract}

\section{Introduction}

The main goal of this paper is to suggest a new construction of infinite finitely generated residually finite torsion groups. First examples of such groups were discovered by Golod [8] and many other constructions have been found since then [2, 9, 11, 16]. Our approach is similar to that from [16. Although it is quite elementary, it allows us to construct first examples of infinite finitely generated residually finite torsion groups with positive rank gradient.

Recall that the rank gradient of a finitely generated group $G$ is defined by the formula

$$
\operatorname{RG}(G)=\inf _{[G: H]<\infty} \frac{d(H)-1}{[G: H]}
$$

where $d(H)$ denotes the minimal number of generators of $H$ and the infimum is taken over all finite index subgroups $H \leq G$. This notion was first introduced by Lackenby in [12] with motivation from 3-dimensional geometry and has received lots of attention since then.

Note that $\operatorname{RG}\left(F_{m}\right)=m-1$ for a free group $F_{m}$ of rank $m$ by the Nielsen-Schreier Formula. In [12, Lackenby proved that $\operatorname{RG}(G)>0$ for every free product $G=A * B$, where at least one of the multiplies is not isomorphic to $\mathbb{Z}_{2}$. On the other hand, many groups have zero rank gradient. This is easy to verify for $S L_{n}(\mathbb{Z})$ if $n \geq 3$, ascending $H N N$-extensions, and direct products of finitely generated infinite residually finite groups [3, 12]. Further $\operatorname{RG}(G)=0$ whenever $G$ is amenable. This was first proved by Lackenby 12 for finitely presented groups and then by Abert and Nikolov in the general case [3].

*This work has been partially supported by the NSF grant DMS-0605093.

Keywords. Torsion group, residually finite group, rank gradient, amenability, $L^{2}$-betti number, cost of a group.

2000 Mathematical Subject Classification. 20F69, 20F05, $20 \mathrm{E} 26$. 
Recall also that, given a (finite or infinite) sequence of primes $\Pi=\left(p_{i}\right)$, a $\Pi$-series in a group $G$ is any chain of normal subgroups

$$
G=G_{0} \triangleright G_{1} \triangleright \ldots,
$$

such that $G_{i-1} / G_{i}$ is either trivial or abelian of exponent $p_{i}$. A group $G$ is $\Pi$-graded if $G$ admits a $\Pi$-series $\left\{G_{i}\right\}$ such that $\bigcap_{i} G_{i}=\{1\}$. Note that all subgroups $G_{i}$ have finite index in $G$ whenever $G$ is finitely generated. In particular, every finitely generated $\Pi$-graded group is residually finite.

In [17, examples of finitely generated infinite torsion $\Pi$-graded groups were constructed for any infinite sequence of primes $\Pi$, but the approach used in [17] did not allow to control rank gradient. In this paper we prove the following.

Theorem 1.1. For every infinite sequence of primes $\Pi$, there exist a finitely generated infinite torsion $\Pi$-graded group with positive rank gradient.

It is easy to show that if $\Pi=(p, p, \ldots)$, then every torsion $\Pi$-graded group is a $p$-group. Thus we obtain the following.

Corollary 1.2. For every prime p, there exist a finitely generated infinite residually finite p-group with positive rank gradient.

Applying Theorem 1.1 to a sequence $\Pi$ of pairwise distinct primes, one can also obtain examples of different nature (see Section 3). It is also worth noting that the finitely generated residually finite torsion $p$-groups constructed by Aleshin [2], Grigorchuck [9] and Gupta-Sidki [11] have zero rank gradient since they are amenable. On the other hand, we do not know whether Golod-Shafarevich groups have positive rank gradient.

Theorem 1.1 is partially motivated by the von Neumann-Day Problem in the class of residually finite groups. Recall that the original problem asks whether there exists a nonamenable group without non-abelian free subgroups. The affirmative answer was obtained by Olshanskii in [15]. Other examples can be found in [1, 10, 17]. However the question remained open for residually finite groups until it was recently solved affirmatively by Ershov [5]. The solution is based on an unexpected example of a Golod-Shafarevich group with property $(\mathrm{T})$ whose construction involves quite complicated arguments. Combining Theorem 1.1 and the theorem of Lackenby-Abert-Nikolov mentioned above allows us to recover Ershov's result.

Corollary 1.3 (Ershov). There exists a non-amenable residually finite group without free subgroups.

The notion of rank gradient is closely related to some other group invariants. Indeed for every finitely generated residually finite group $G$, we have

$$
\operatorname{RG}(G) \geq \operatorname{cost}(G)-1 \geq \beta_{1}^{(2)}(G)-\frac{1}{|G|}
$$

where $\operatorname{cost}(G)$ denotes the cost of $G, \beta_{1}^{(2)}(G)$ is the first $L^{2}$-Betti number of $G$ (we refer to [7, 14] for the definitions), and $1 /|G|=0$ if $G$ is infinite. The first inequality in (2) is due 
to Abert and Nikolov [4] and the second one is due to Gaboriau [6]. In fact, in all cases where the the exact values of these quantities are calculated they are equal, and it is still unknown whether the inequalities in (2) can be strict. The following two questions were asked by Gaboriau [7, 6].

Problem 1.4 (Fixed Price). Does every finitely generated group have fixed price?

Recall that a countable group $G$ has fixed price, if all essentially free measure-preserving Borel actions of $G$ have the same cost, which equals $\operatorname{cost}(G)$ in this case. Abert and Nikolov [4] proved that for every finitely generated residually finite group $G, \operatorname{RG}(G)$ equals the cost of the natural action of $G$ on its profinite completion endowed with the Haar measure minus 1. Thus the Fixed Price Conjecture would imply the equality $\operatorname{RG}(G)=\operatorname{cost}(G)-1$.

Problem 1.5 (First $L^{2}$-Betti Number vs. Cost). Is $\operatorname{cost}(G)-1=\beta_{1}^{(2)}(G)$ for every finitely generated infinite group $G$ ?

The first $L^{2}$-Betti number of a finitely presented residually finite group $G$ can be defined in purely group theoretic terms due to the following Approximation Theorem of Luck [14. Let $\left\{N_{i}\right\}$ be a nested sequence of finite index normal subgroups of $G$ with trivial intersection. Then we have

$$
\beta_{1}^{(2)}(G)=\lim _{i \rightarrow \infty} \frac{b_{1}\left(N_{i}\right)}{\left[G: N_{i}\right]},
$$

where $b_{1}\left(N_{i}\right)$ is the ordinary first Betti number of $N_{i}$. However the answer to the following question is still unknown.

Problem 1.6 (Approximation for Finitely Generated Groups). Does the approximation (3) hold for every finitely generated residually finite group $G$ ?

It is easy to see that Theorem 1.1 implies the following.

Corollary 1.7. At least one of the Problems 1.41.6 has negative solution.

Indeed let $G$ be a finitely generated residually finite torsion group with positive rank gradient. We obviously have $b_{1}(N)=0$ for every subgroup $N \leq G$ and hence the right side of (3) equals zero. Thus either (3) is violated or at least one of the inequalities in (2) is strict.

Acknowledgments. I am grateful to Miklos Abert and Alexander Olshanskii for useful discussions. I am also grateful to Misha Ershov who suggested Lemma [2.6 and noticed that it can be used to show that finitely generated infinite residually finite $p$-groups constructed by our method have positive rank gradient. This was overlooked by the author in the first version of the paper.

\section{Preliminaries}

In this section we collect some auxiliary definitions and results used in the proof of our main theorem. Given a group $G$ and elements $x, y \in G$, we write $x^{y}$ for $y^{-1} x y$. We denote by 
$\langle\langle S\rangle\rangle^{G}$ (or just $\langle\langle S\rangle$ if no confusion is possible) the normal closure of a subset $S$ in $G$, i.e., the smallest normal subgroup of $G$ containing $S$. By $d(G)$ we denote the minimal number of generators of a finitely generated group $G$. Finally if $G$ is finitely presented, $\operatorname{def}(G)$ denotes the deficiency of $G$.

Given a finitely presented group $G$, its deficiency is defined as the maximum of $d-r$ over all finite presentations

$$
G=\left\langle x_{1}, \ldots, x_{d} \mid R_{1}, \ldots, R_{r}\right\rangle
$$

We start with an obvious observation. It is an immediate corollary of the NeilsenSchreier formula.

Lemma 2.1. Let $G$ be a finitely generated group, $K \leq H$ finite index subgroups of $G$. Then $(d(K)-1) /[G: K] \leq(d(H)-1) /[G: H]$.

The next lemma is also well-known. We outline the proof for the sake of completeness.

Lemma 2.2. Let $G$ be a finitely presented group, $H$ a finite index subgroup of $G$. Then $\operatorname{def}(H)-1 \geq(\operatorname{def}(G)-1)[G: H]$.

Proof. Let $G=F / R$, where $F=\left\langle x_{1}, \ldots, x_{d}\right\rangle$ is free of rank $d, R=\left\langle\left\langle R_{1}, \ldots, R_{r}\right\rangle\right\rangle^{F}$, and $\operatorname{def}(G)=d-r$. By the Nilsen-Schreier Formula, the full preimage $K$ of $H$ in $F$ has rank $(d-1) j+1$, where $j=[F: K]=[G: H]$. It is straightforward to check that $R=\left\langle\left\langle R_{i}^{t}, i=1, \ldots, r, t \in T\right\rangle\right\rangle^{K}$, where $T$ is a set of left coset representatives of $K$ in $F$. Thus $H=K / R$ has a presentation with $(d-1) j+1$ generators and $r|T|=r j$ relations, which implies

$$
\operatorname{def}(H)-1 \geq(d-1) j-r j=(d-r-1) j=(\operatorname{def}(G)-1)[G: H] .
$$

Lemma 2.3. Let $G$ be a finitely presented group, $N$ a finite index normal subgroup of $G$, $g$ an element of $G, m$ the order of $g N$ in $G / N$. Let $M$ denote the natural image of $N$ in the quotient group $Q=G /\left\langle\left\langle g^{m}\right\rangle\right.$. Then $\operatorname{def}(M) \geq \operatorname{def}(N)-[G: N] / m$.

Proof. Let $T$ denote the set of representatives of right cosets of $\langle g\rangle N$ in $G$. It is an easy exercise to prove that $\langle\langle g\rangle\rangle^{G}=\langle\langle Z\rangle\rangle^{N}$, where $Z=\left\{g^{t} \mid t \in T\right\}$ (see, e.g., [17][Lemma 2.3]). Thus given a presentation of $N$, we can get a presentation of $M$ by adding $|T|=[G:\langle g\rangle N]=$ $[G / N:\langle g\rangle N / N]=[G: N] / m$ relations. This implies the statement of the lemma.

To each group $G$ and each sequence of primes $\Pi=\left(p_{i}\right)$, we associate a sequence of subgroups $\left\{\delta_{i}^{\Pi}(G)\right\}$ of $G$ defined by $\delta_{0}^{\Pi}(G)=G$ and

$$
\delta_{i}^{\Pi}(G)=\left[\delta_{i-1}^{\Pi}(G), \delta_{i-1}^{\Pi}(G)\right]\left(\delta_{i-1}^{\Pi}(G)\right)^{p_{i}} .
$$

It is easy to prove by induction that these subgroups form a $\Pi$-series. Thus a group $G$ is $\Pi$-graded if and only if

$$
\bigcap_{i=1}^{\infty} \delta_{i}^{\Pi}(G)=\{1\} .
$$


The proof of the following lemma is elementary and can be found in [16, Lemma 3.3 a)].

Lemma 2.4. Let $\Pi=\left(p_{1}, p_{2}, \ldots\right)$ be an infinite sequence of primes, $G$ a torsion $\Pi$-graded group. Then for every $n \in \mathbb{N}$, every element of $\delta_{n}^{\Pi}(G)$ has order $p_{n+1}^{\alpha_{n+1}} \cdots p_{n+k}^{\alpha_{n+k}}$ for some integers $\alpha_{n+1}, \ldots, \alpha_{n+k}$ and $k>0$.

Corollary 2.5. Let $\Pi$ be an infinite sequence of pairwise distinct primes, $G$ a $\Pi$-graded torsion group. Then every finite index subgroup $H \leq G$ contains $\delta_{n}^{\Pi}(G)$ for some $n$.

Proof. Since every finite index subgroup contains a finite index normal subgroup, we may assume that $H$ is normal without loss of generality. Let $\Pi=\left(p_{1}, p_{2}, \ldots\right)$. As all primes in $\Pi$ are distinct, there exists an integer $n$ such that $|G / H|$ is not divisible by $p_{j}$ for any $j>n$. Let $x$ be an arbitrary element of $\delta_{n}^{\Pi}(G)$. By Lemma 2.4 we have $\operatorname{gcd}(|x|,|G / H|)=1$ and hence the image of $x$ in $G / H$ is trivial. Thus we have $\delta_{n}^{\Pi}(G) \leq H$.

The following analogue of Corollary 2.5 for $\Pi=(p, p, \ldots)$, where $p$ is a prime, is quite trivial.

Lemma 2.6. Let $\Pi=(p, p, \ldots)$, where $p$ is a prime, and let $G$ be a torsion $\Pi$-graded group. Then every finite index subgroup of $G$ contains $\delta_{n}^{\Pi}(G)$ for some $n$.

Proof. Let $H \leq G$, where $G$ is $\Pi$-graded and $H$ is of finite index. Again we may assume that $H$ is normal in $G$. Then $G$ is a $p$-group according to Lemma 2.4 and hence so is $Q=G / H$. Since every finite $p$-group is nilpotent, we have $\delta_{1}^{\Pi}(Q)=[Q, Q] Q^{p} \neq Q$. Therefore $\delta_{n}^{\Pi}(Q)=1$ for some $n$. This implies $\delta_{n}^{\Pi}(G) \leq H$.

\section{Torsion groups with positive rank gradient}

Theorem 1.1 is a particular case of the following.

Theorem 3.1. For every finitely presented group $G$ of deficiency $\operatorname{def}(G) \geq 2$, every infinite sequence of primes $\Pi=\left(p_{1}, p_{2}, \ldots\right)$, and every $\varepsilon>0$, there exists an infinite $\Pi$-graded torsion quotient group $Q$ of $G$ such that

$$
\operatorname{RG}(Q) \geq \operatorname{def}(G)-1-\varepsilon .
$$

Proof. We fix a sequence of primes $\Pi$ and $\varepsilon>0$. Let $r=\operatorname{def}(G)-1-\varepsilon$. Without loss of generality we can assume that $\varepsilon<1$ and hence $r>0$. We enumerate all elements of $G=\left\{g_{0}=1, g_{2}, \ldots\right\}$ and proceed by induction.

Suppose that for some $k \geq 0$, we have already constructed a sequence of epimorphisms

$$
G=G_{0} \stackrel{\alpha_{7}}{\rightarrow} G_{1} \stackrel{\alpha_{2}}{\rightarrow} \ldots \stackrel{\alpha_{k}}{\rightarrow} G_{k}
$$

and a sequence of positive integers $0=n_{0}<n_{1}<\ldots<n_{k}$. In what follows, we denote $\delta_{n_{i}}^{\Pi}\left(G_{i}\right)$ by $D_{i}$. Given a group $H$, we define $\widehat{H}=H / \bigcap_{j=1}^{\infty} \delta_{j}^{\Pi}(H)$. Assume that for every $i=0, \ldots, k$, the following conditions hold. 
(a) The natural image $g_{i}$ in $\widehat{G}_{i}$ has finite order.

(b) $\operatorname{def}\left(D_{i}\right)-1>r\left[G_{i}: D_{i}\right]$.

Further if $k>0$, we assume that

(c) $\operatorname{Ker}\left(\alpha_{i+1}\right) \leq\left[D_{i}, D_{i}\right] D_{i}^{p_{n_{i}+1}}$ for every $i=0, \ldots, k-1$.

The following observation will be used several times below. Suppose that the image of an element $h$ of a group $H$ has finite order in $\widehat{H}$. Then for every quotient group $K$ of $H$, the image of $h$ in $\widehat{K}$ has finite order. This follows immediately from the fact that $\delta_{i}^{\Pi}(H)$ is a verbal subgroup of $H$ and hence $\delta_{i}^{\Pi}(H)=\delta_{i}^{\Pi}(K)$ for all $i$.

Let us consider two cases.

Case 1. Suppose that the natural image of $g_{k+1}$ in $\widehat{G}_{k}$ has finite order. Then we set $G_{k+1}=G_{k}, \alpha_{k+1}=i d$, and $n_{k+1}=n_{k}+1$. Condition (b) for $i=k+1$ easily follows from the inductive assumption and Lemma 2.2 applied to the groups $D_{k+1} \leq D_{k}$. Verifying (a) and (c) is straightforward.

Case 2. Suppose now that the natural image of $g_{k+1}$ in $\widehat{G}_{k}$ has infinite order. This means that the order of the image of $g_{k+1}$ in $G_{k} / \delta_{j}^{\Pi}\left(G_{k}\right)$ tends to $\infty$ as $j \rightarrow \infty$. Therefore by (b) we can find $n_{k+1}>n_{k}$ such that such that

$$
\frac{\operatorname{def}\left(D_{k}\right)-1}{\left[G_{k}: D_{k}\right]}>r+\frac{1}{m},
$$

where $m$ is the order of the image of $g_{k+1}$ in $G_{k} / \delta_{n_{k+1}}^{\Pi}\left(G_{k}\right)$. We set $G_{k+1}=G_{k} /\left\langle\left\langle\bar{g}_{k+1}^{m}\right\rangle\right\}^{G_{k}}$, where $\bar{g}_{k+1}$ is the image of $g_{k+1}$ in $G_{k}$, and let $\alpha_{k+1}$ be the natural homomorphism $G_{k} \rightarrow$ $G_{k+1}$.

Again verifying (c) for $i=k$ is trivial. Indeed we have

$$
\operatorname{Ker}\left(\alpha_{k+1}\right)=\left\langle\left\langle\bar{g}_{k+1}^{m}\right\rangle\right\rangle^{G_{k}} \leq \delta_{n_{k+1}}^{\Pi}\left(G_{k}\right) \leq \delta_{n_{k}+1}^{\Pi}\left(G_{k}\right)=\left[D_{k}, D_{k}\right] D_{k}^{p_{k+1}}
$$

since $\bar{g}_{k+1}^{m} \in \delta_{n_{k+1}}^{\Pi}\left(G_{k}\right)$ by our construction.

It is also straightforward to verify (a) for $i=k+1$. Finally to prove (b) we note that $D_{k+1}=\delta_{n_{k+1}}^{\Pi}\left(G_{k+1}\right)=\alpha_{k+1}\left(\delta_{n_{k+1}}^{\Pi}\left(G_{k}\right)\right)$. Using subsequently Lemma 2.3, Lemma 2.2. (8) , and the inclusion $\operatorname{Ker}\left(\alpha_{k+1}\right) \leq \delta_{n_{k+1}}^{\Pi}\left(G_{k}\right)$, we obtain

$$
\begin{aligned}
\operatorname{def}\left(D_{k+1}\right)-1 \geq & \operatorname{def}\left(\delta_{n_{k+1}}^{\Pi}\left(G_{k}\right)\right)-1-\frac{1}{m}\left[G_{k}: \delta_{n_{k+1}}^{\Pi}\left(G_{k}\right)\right] \geq \\
& \left(\operatorname{def}\left(D_{k}\right)-1\right)\left[D_{k}: \delta_{n_{k+1}}^{\Pi}\left(G_{k}\right)\right]-\frac{1}{m}\left[G_{k}: \delta_{n_{k+1}}^{\Pi}\left(G_{k}\right)\right]> \\
& \left(r+\frac{1}{m}\right)\left[G_{k}: D_{k}\right]\left[D_{k}: \delta_{n_{k+1}}^{\Pi}\left(G_{k}\right)\right]-\frac{1}{m}\left[G_{k}: \delta_{n_{k+1}}^{\Pi}\left(G_{k}\right)\right]= \\
& r\left[G_{k}: \delta_{n_{k+1}}^{\Pi}\left(G_{k}\right)\right]= \\
& r\left[\alpha_{k+1}\left(G_{k}\right): \alpha_{k+1}\left(\delta_{n_{k+1}}^{\Pi}\left(G_{k}\right)\right)\right]= \\
& r\left[G_{k+1}: D_{k+1}\right] .
\end{aligned}
$$


This finishes the inductive step.

Let now $P$ be the limit group. That is, let $P=G_{0} / \bigcup_{i=1}^{\infty} K_{i}$, where $K_{i}=\operatorname{Ker}\left(\alpha_{i} \circ \cdots \circ\right.$ $\left.\alpha_{1}\right)$. Further let $Q=\widehat{P}$. Clearly $\bigcap_{i=1}^{\infty} \delta_{i}^{\Pi}(Q)=\{1\}$ and hence $Q$ is a $\Pi$-graded group. It follows from (a) that every element of $Q$ has finite order.

Let

$$
A_{i}=\delta_{n_{i}}^{\Pi}(Q) /\left[\delta_{n_{i}}^{\Pi}(Q), \delta_{n_{i}}^{\Pi}(Q)\right]\left(\delta_{n_{i}}^{\Pi}(Q)\right)^{p_{n_{i}+1}} .
$$

Observe that for every $i \in \mathbb{N}, A_{i}$ is isomorphic to $D_{i} /\left[D_{i}, D_{i}\right] D_{i}^{p_{n_{i}+1}}$ as $\operatorname{Ker}\left(G_{i} \rightarrow Q\right) \leq$ $\left[D_{i}, D_{i}\right] D_{i}^{p_{n_{i}+1}}$ by (c). Hence $A_{i}$ is isomorphic to a quotient group of $\left(\mathbb{Z} / \mathbb{Z}_{p_{n_{i}+1}}\right)^{d_{i}}$ modulo a subgroup of rank $r_{i}$, where $d_{i}-r_{i}=\operatorname{def}\left(D_{i}\right)$. In particular, $d\left(A_{i}\right) \geq \operatorname{def}\left(D_{i}\right)$ and using (b) we obtain

$$
d\left(\delta_{n_{i}}^{\Pi}(Q)\right)-1 \geq d\left(A_{i}\right)-1 \geq \operatorname{def}\left(D_{i}\right)-1>r\left[G_{i}: D_{i}\right]=r\left[Q: \delta_{n_{i}}^{\Pi}(Q)\right]
$$

for every $i \in \mathbb{N}$. Again we consider two cases.

Case 1. Suppose that $\Pi$ contains infinitely many distinct primes. Note that if a group is $\Xi$-graded for some subsequence $\Xi$ of $\Pi$, then it is $\Pi$-graded as well. Thus passing to a subsequence of $\Pi$ if necessary we can assume that $\Pi$ consists of pairwise distinct primes. Let $Q$ be an infinite $\Pi$-graded torsion quotient group of $G$ satisfying (9). For every finite index subgroup $H \leq Q$, we have $(d(H)-1) /[Q: H] \geq r$ by Corollary 2.5, Lemma 2.1, and (9). Thus $\operatorname{RG}(Q) \geq r=\operatorname{def}(G)-1-\varepsilon$.

Case 2. If $\Pi$ contains finitely many distinct primes, then at least one prime $p$ occurs in $\Pi$ infinitely many times. Thus passing to a subsequence if necessary we can assume that $\Pi=(p, p, \ldots)$. The rest of the proof in this case is the same as above. The only difference is that we have to use Lemma 2.6 instead of Corollary 2.5.

Proof of Corollary 1.2. Let $\Pi=(p, p, \ldots)$. By Theorem 3.1 there exists a finitely generated residually finite infinite $\Pi$-graded torsion group $Q$ with positive rank gradient. By Lemma $2.4 Q$ is a $p$-group.

Corollary 1.3 is a particular case of the following.

Corollary 3.2. For every infinite sequence of primes $\Pi$, there exists a finitely generated torsion non-amenable $\Pi$-graded group $G$. In particular, for every prime $p$, there exists a finitely generated residually finite non-amenable p-group.

Proof. By the Lackenby-Abert-Nikolov Theorem [3], the groups constructed in Theorem 3.1] are non-amenable if $\varepsilon<1$.

\section{References}

[1] S.I. Adian, Random walks on free periodic groups, Izv. Akad. Nauk SSSR, Ser. Mat., 46(1982), 6, 1139-1149. 
[2] N. V. Aleshin, Finite automata and the Burnside problem for periodic groups, (Russian) Mat. Zametki 11 (1972), 319-328.

[3] M. Abert, N. Nikolov, The rank gradient from a combinatorial viewpoint, arXiv:math/0701925.

[4] M. Abert, N. Nikolov, Rank gradient, cost of groups and the rank versus Heegaard genus problem, arXiv:math/0701361.

[5] M. Ershov, Golod-Shafarevich groups with property $(T)$ and Kac-Moody groups, Duke Math. J. 145 (2008), 2, 309-339.

[6] D. Gaboriau, Invariants $l^{2}$ de relations d'équivalence et de groupes, Publ. Math. IHES 95 (2002), 93-150.

[7] D. Gaboriau, Coût des relations d'équivalence et des groupes, Invent. Math. 139 (2000), $1,41-98$.

[8] E.S. Golod, On nil-algebras and finitely approximable $p$-groups (Russian), Izv. Akad. Nauk SSSR, Ser. Mat. 28 (1964), 273-276.

[9] R. I. Grigorchuk, On Burnside's problem on periodic groups, Functional Anal. Appl. 14 (1980), no. 1, 41-43.

[10] M. Gromov, Hyperbolic groups, in: Essays in Group Theory (S.M.Gersten, ed.), M.S.R.I. Pub. 8, Springer, 1987, 75-263.

[11] N. Gupta, S. Sidki, On the Burnside problem for periodic groups, Math. Z. 182 (1983), $385-388$.

[12] M. Lackenby, Expanders, rank and graphs of groups, Israel J. Math. 146 (2005), 357370.

[13] G. Levitt, On the cost of generating an equivalence relation, Ergodic Theory Dynam. Systems 15 (1995), 6, 1173-1181.

[14] W. Lück, $L^{2}$-invariants: theory and applications to geometry and $K$-theory. A Series of Modern Surveys in Mathematics, 44. Springer-Verlag, Berlin, 2002.

[15] A. Olshanskii, On the question of the existence of an invariant mean on a group, (Russian) Uspekhi Mat. Nauk 35 (1980), 4, 199-200.

[16] A.Yu. Olshanskii, D. Osin, Large groups and their periodic quotients, Proc. Amer. Math. Soc 136 (2008), 753-759.

[17] A. Olshanskii, M. Sapir, Non-amenable finitely presented torsion-by-cyclic groups, Publ. IHES. 96 (2002), 43-169. 